\title{
Cocoon: Cooperative Sensorcommunication - a new approach towards Wireless Communication in Sensor Networks
}

\author{
A. Buchmann ${ }^{1}$, D. Dahlhaus ${ }^{2}$, M. Pesavento ${ }^{1}$, K. Hofmann ${ }^{1}$ M. Hollick ${ }^{1}$, R. Jakoby ${ }^{1}$, A. Klein ${ }^{1}$, \\ S. Santini ${ }^{1}$, S. Ulbrich ${ }^{1}$, T. Weiland ${ }^{1}$, A. Zoubir ${ }^{1}$ \\ ${ }^{1}$ TU Darmstadt, Germany, ${ }^{2}$ :U Kassel, Germany \\ Klaus.Hofmann@ies.tu-darmstadt.de
}

\begin{abstract}
Innovation in wireless communication enables the majority of the world's population to use mobile phones and wireless networks. Ubiquitous communication requires sophisticated networks, wellconnected mobile devices, progress in base-technologies and communication paradigms. The LOEWE priority program Cocoon (Cooperative Sensorcommunication, funded by the state of Hesse, Germany) puts an emphasis on comprehensive research in the field of networks, signal processing and reconfigurable transceiver architectures, and aims to verify the usability of these communication paradigms in Smart Cities and Car-to-X communication.
\end{abstract}

Key words: Networks, Sensor Communication, Signal Processing, Reconfigurable Transceiver, Smart Cities

\section{Structure of the Cocoon project}

The authors of this paper are the principal investigators of the LOEWE (L्andes-Offensive zur Entwicklung Wissenschaftlich-ökonomischer Exzzellenz) Research Priority Program "Cooperative sensor communication = Cocoon". The project has been established January 2011 and is funded with an amount of 4.5 Mio. $€$ for a period of 3 years by the State of Hesse. With this paper an overview on the research direction and achievements will be given.

The Cocoon project is structured in four major working areas:

- A: Networking Aspects

- B: Signal Processing

- C: Transceiver Architectures

- D: Demonstrator and Verification

\section{Working Area A: Networking Aspects}

The main goals of working area $A$ were to conduct a requirements analysis and to identify adequate optimization criteria for the two focus topics areas wireless sensor networks and wireless meshed networks. Moreover, first mechanism and protocol designs were to be designed.

In the area of wireless sensor networks, the groups of Prof. Buchmann and Santini worked towards defining the requirements of an adaptive, component-based framework for monitoring wireless multihop networks.

A thorough survey of the state-of-the-art allowed identifying basic metrics and mechanisms required to provide a continuous, reliable monitoring of network performance.

Moreover, concrete mechanisms have been proposed to allow for efficient coding of eventbased communications and of device capabilities. In particular, the ASN.1 standard has been identified as a matching standard to define information exchanged by heterogeneous devices and described the message contents. The corresponding Packed Encoding Rules (ASN.1 PER) can be used to encode/decode the information to/from bytestream in byte-length efficient way for transmission. For the description of different capabilities of the devices a predefined classification has been suggested which can be extended on purpose.

In the area of communication mechanisms for wireless mesh and sensor networks, the groups of Prof. Klein and Prof. Hollick have been extensively studying novel algorithms suitable for applications in large-scale sensor and mesh networks.

In particular, wireless sensor networks that concurrently support multiple applications with different computation functions have been studied. Novel two-way relaying mechanisms, 
which support bi-directional communications between sensor nodes, and that perform computation on the physical layer by means of coding, have been designed. It has been shown by simulation that the superposition coding protocol can achieve better sum-rate performance than existing time-division protocols.

Novel cross-layer communication mechanisms have been designed to support OFDMA for wireless multihop networks. In particular, various dynamic subchannel allocation schemes for OFDMA-based wireless mesh networks have been proposed and evaluated by means of simulation. As a proof-of-concept, selected schemes have been implemented using the WARP software-defined radio platform.

\section{Working Area B: Signal Processing}

Inspired by a distributed pressure sensing application formulated by the industrial partner Hydrotechnik $\mathrm{GmbH}$, a power efficient physical layer consensus algorithm was developed that fully exploits the broadcast character of the wireless channel to compute the average pressure in the network in a fully distributed manner. In a cooperation between the Communication System Group and the Research Group Nonlinear Optimization the problem of network optimization in small size cellular networks with Coordinated Multipoint (CoMP) transmission has been addressed, where the beamforming weights and power allocations are jointly optimized together with the network topologies using a mixed-integer programming framework. In this cooperation and using a similar approach we have also considered optimal beamforming and rate adaptation for next generation cellular systems like LTE and LTE-Advanced.

To address the need for increased data rate, multiple transmitter receiver pairs communicate simultaneously utilizing the same resources. The simultaneous transmission leads to large amount of interferences. Based on the principle of interference alignment, a relay aided cooperative beamforming scheme has been developed to achieve interference free communication.

In another project, there was an investigation of single cell and ad-hoc scenarios with multiple source-destination pairs which bidirectionally communicate with each other via a multiantenna relay station. The focus was on developing low-complexity spatial two-way relay transceive filters and transmission schemes to support asymmetric data rates in these scenarios.
Furthermore, work has been focused on distributed detection, sequential analysis and diffusion algorithms. So far, the following results were obtained: Under Bayesian uncertainty, we established that the loss in terms of probability of error between robust and minimum error detection is bounded. The results were extended to a general model of heavy-tailed priors, the so-called epsilon-contaminated model and the corresponding bounds were derived. Both results, initially obtained for a single sensor, were then extended to the multisensor case where a fusion center combines the decisions of each single sensor in a decentralized manner.

Further investigation was done concerning the influence of correlated data on sequential detection and, in particular, quickest change detection. The case of sequentially detecting correlated signals in Gaussian noise was studied in more detail and asymptotic results and performance bounds were derived. Interestingly, while the theoretical bounds on the detection delay of sequential tests usually increase with correlation, the opposite is true for detecting.

In the context of adaptive cooperative wireless sensor networks, diffusion adaptive algorithms were investigated and implemented. These are distributed algorithms that can be tailored to a host of applications typical of wireless sensor networks. Of specific interest are diffusion adaptive algorithms for inference, such as detection and estimation. Their advantage is their applicability in networks where sensors rely solely on local interactions and in-network processing, and are able, on the fly, to learn environmental statistics, and adapt their dynamics to changing conditions.

Moreover, a simple relay architecture with realistic assumptions for interference mitigation using distributed beamforming was developed. The architecture is less complex and provides an acceptable performance compared with the optimum scheme. In the context of Filter Bank Based Reconfigurable Receiver Architecture, a reconfigurable receiver architecture building on concepts from time-frequency analysis was designed. It can be used, for instance, for DSSS signaling based UMTS and OFDM based WLANs. Furthermore, a numerical procedure for DFT filter banks used for spectrum sensing in cognitive radio was designed. It reduces the spectrum estimation complexity compared with the optimum scheme.

\section{Working Area C: Transceiver Architectures}

Area $C$ investigates reconfigurable transceiver architectures in combination with reconfigurable 
baseband processing from area D. A demonstrator is made of several individual modules (tunable antenna, digital signal processing FPGA, high-voltage single chip DC/DC converter and control circuitry), see also Figure 1.

The first realization of the system should cover the ISM-bands at $\mathrm{f}_{0}=2.45 \mathrm{GHz}, 5.1 \mathrm{GHz}$, and $5.8 \mathrm{GHz}$ with a maximum RF-bandwidth of BRF $\leq 10 \mathrm{MHz}$ and sampling rate of $\mathrm{f}_{\mathrm{s}} \leq 40 \mathrm{MHz}$ with 10 bit resolution. The components for the tunable antenna module are under development. For the biasing of the tunable BST thick-film varactors of the antenna a high voltage up to $\mathrm{V}_{\mathrm{t}, \max }=120 \mathrm{~V}$ is required. Therefore a monolithic integrated DC/DCconverter and a monolithic integrated variable voltage source are developed for the $0.35 \mu \mathrm{m}$ HV-CMOS process from austriamicrosystems (AMS H35) implementing a novel four-phase clock scheme. The die has a size of $3.9 \times 5.6 \mathrm{~mm}^{2}$ and requires no external components. With $3.7 \mathrm{~V}$ supply as provided from batteries, $10 \mathrm{MHz}$ clock frequency, $\mathrm{R}_{\text {load }}=1.05 \mathrm{M} \Omega, \mathrm{C}_{\text {load }}=30 \mathrm{pF}$ an output voltage of $116.1 \mathrm{~V}$ could be achieved in post-layout simulations including clock buffers. Next steps are the combination of the different modules built at TU Darmstadt and Uni Kassel to one demonstration system which can be used by the consortium for evaluation. This system will combine the hardware as well as the control software which adapts the frontend properties automatically to the requirements of the signal to be transmitted. The modulation/demodulation of the signals will be conducted by commercial systems from Agilent Technologies (MXA, MGA) for a fast demonstrator realization. In further steps these systems could be replaced by own components towards a single transceiver board. Also duplex and semi-duplex operation is planned for further steps. The design of intelligent antenna systems is nowadays based on full-wave electromagnetic simulation tools. Due to the complexity of the underlying model a large amount of degrees of freedom has to be considered in the numerical evaluation process. To enable the desired simulation of the entire device on the circuit level including the real antenna system a reduced model has to be introduced. In the current work both requirements are incorporated into a so-called parameterdepending reduced model while the original parameter dependencies are also retained in the reduced circuit model. The simplified model can be used instead of the original model in a large variety of applications. The implemented algorithms have been tested on characteristic filter applications where full-wave reference solutions can still be obtained. The focus of the current research is put on the development of efficient algorithms which support geometrical variations within individual discretization cells. This kind of variability can immediately be applied to provide a robust sensitivity analysis even on the circuit level.

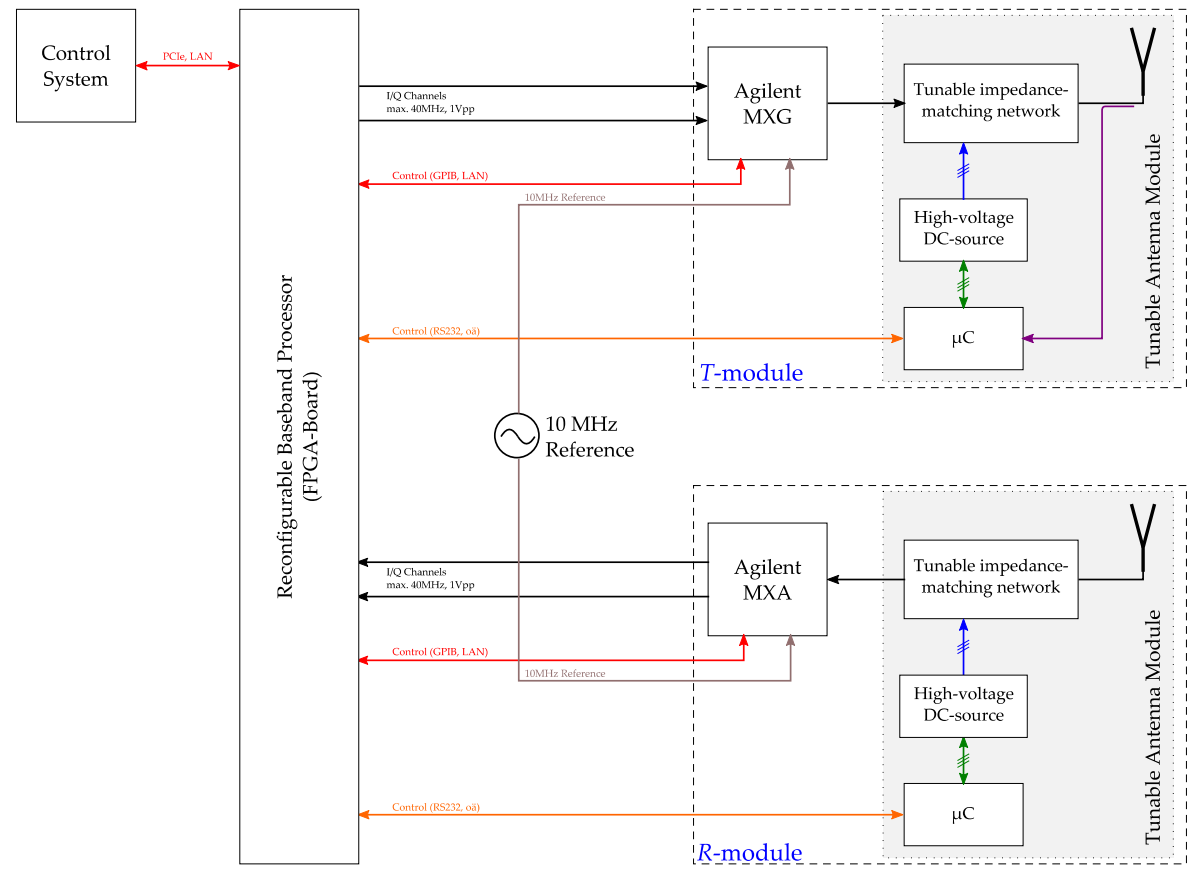

Fig. 1. Reconfigurable Transceiver Architecture 


\section{Working Area D: Demonstrator and Verification}

Working Area $D$ aims to bundle the results from areas A-C and verify the usability of the individual achievement in the frame of chosen demonstrators.

- A testbed consisting of 4 WARP softwaredefined radio nodes has been successfully established. A dynamic subchannel allocation scheme for OFDMA-based wireless mesh networks has been proposed and implemented using the WARP platform. The correct working of the mechanisms has been validated by means of experimentation. Further optimization and study is planned for within the 2012 workplan.

- In order to harvest the merits of coordinated multi-point processing while keeping the associated operational costs acceptable, we consider the problem of joint multicell base station assignment and beamformer design. The problem is addressed using mixed integer second order cone programming. An improved reformulation of the mathematical model as well as heuristics were developed to improve the solution process using branch-and-bound type methods.

- To enhance the throughput in multi-hop sensor networks, a corridor-based routing scheme using opportunistic forwarding has been developed. In contrast to conventional unipath routing where the transmission of the data is forced to follow a fixed sequence of nodes, we widen this path to a corridor consisting of a certain number of forwarding nodes along the route. Inside this corridor, data can be split and joined as it travels towards the destination node. To split data at a given node, Orthogonal Frequency Division Multiple Access (OFDMA) is used since OFDMA offers the opportunity of allocating different subcarriers to different nodes according to their channel conditions, i.e., information from the physical layer can be incorporated into the network layer unipath route in a cross-layer manner. Simulations showed that corridorbased routing outperforms conventional unipath routing approaches in terms of achievable throughput.

A reconfigurable transceiver has been specified and programmed in a MATLAB simulation environment. Different parameters of the transceiver, e.g. the modulation of the chosen transmission standard defined by the chosen medium-access control (MAC), can be accounted for by the pulse optimization scheme. Further interfaces are to be defined between the MAC and the physical layer (PHY) to extend the transceiver functionalities beyond the ones in area $C$ to investigate constraints from e.g. real-time processing or hardware limitations.

\section{Summary}

The Cocoon project has been started in Jan 2011 and will continue until December 2013. By that time, we will have achieved most objectives set at the start of the project. As it is usually the case new research questions and approaches have been found during any research project. The team will therefore strive to build a larger and more interdisciplinary consortium to realize the Cocoon research findings and start timely most challenging projects which will enable the realization of the vision of a smart city.

\section{References}

[1] H. Frey, M. Hollick, A. Loch: "Curve-based Planar Graph Routing with Guaranteed Delivery in Multihop Wireless Networks", In Proceedings of the 13th IEEE International Symposium on a World of Wireless, Mobile and Multimedia Networks (WoWMoM 2012), 2012

[2] P. Guerrero, A. Buchmann, A. Khelil, K. Van Laerhoven. "TUD $\mu$ Net, a Metropolitan-Scale Federation of Wireless Sensor Network Testbeds", Proceedings of the 9th EWSN conference, Trento, Feb. 2012

[3] P. Guerrero, A. Buchmann, K. Van Laerhoven, I. Schweizer, M. Mühlhäuser, T. Strufe, S. Schneckenburger, M. Hegger, B. Kretzschmar: "A Metropolitan-Scale Testbed for Heterogeneous Wireless Sensor Networks to Support CO2 Reduction", 2nd GreeNets, Spain, ICST, October 2012

[4] P. Guerrero, I. Gurov, A. Buchmann, K. Van Laerhoven, "Diagnosing the Weakest Link in WSN Testbeds: A Reliability and Cost Analysis of the USB Backchannel", 7th IEEE SenseApp, Clearwater, FL, USA, Oct. 2012

[5] Christian Beckel, Leyna Sadamori, Silvia Santini: "Towards Automatic Classification of Private Households Using Electricity Consumption Data". In: Proceedings of the 4th ACM Workshop on Embedded Sensing Systems for EnergyEfficiency in Buildings (BuildSys 2012). November 2012

[6] Andreas Reinhardt, Paul Baumann, Daniel Burgstahler, Matthias Hollick, Hristo Chonov, Marc Werner, Ralf Steinmetz: "On the Accuracy of Appliance Identification Based on Distributed Load Metering Data". In: Proceedings of the 2nd 
IFIP Conference on Sustainable Internet and ICT for Sustainability (SustainIT), October 2012

[7] Christian Beckel, Wilhelm Kleiminger, Thorsten Staake, Silvia Santini: "Improving device-level electricity consumption breakdowns in private households using ON/OFF events", in ACM SIGBED Review - Special Issue on the 3rd International Workshop on Networks of Cooperating Objects (CONET 2012), Vol. 9, No . 3, ACM, pp. 32-38, New York, NY, USA, July 2012

[8] Andreas Reinhardt, Olivia Morar, Silvia Santini, Sebastian Zöller, Ralf Steinmetz: "CBFR: Bloom Filter Routing with Gradual Forgetting for Treestructured Wireless Sensor Networks with Mobile Nodes". In: Proceedings of the IEEE International Symposium on a World of Wireless Mobile and Multimedia Networks (WoWMoM 2012). June 2012

[9] Christian Beckel, Wilhelm Kleiminger, Thorsten Staake, Silvia Santini: "Improving Device-level Electricity Consumption Breakdowns in Private Households Using ON/OFF Events". In: Proceedings of the 3rd International Workshop on Networks of Cooperating Objects (CONET 2012). Co-located with the CPS Week 2012. April 2012

[10] Z. Chen, A. Kühne, A. Klein, „Delay Constraints for Multiple Applications in Wireless Sensor Networks," in Proc. International Symposium on Wireless Communication Systems (ISWCS'12), Paris, France, August 2012

[11] Z. Chen, A. Kühne, A. Klein, A. Loch, M. Hollick and J. Widmer, „Two-Way Relaying for Multiple Applications in Wireless Sensor Networks," in Proc. International ITG Workshop on Smart Antennas (WSA'12), Dresden, Germany, March 2012

[12] A. Kühne and A. Klein, „Hybrid adaptive/nonadaptive multi-user OFDMA systems in the presence of user-specific imperfect channel knowledge," EURASIP Journal on Wireless Communications and Networking 2012, 2012:207, July 2012

[13] A. Kühne, A. Klein, A. Loch and M. Hollick, „Corridor-based routing using opportunistic forwarding in multi-hop OFDMA networks," in Proc. International Symposium on Personal, Indoor and Mobile Radio Communications (PIMRC'12), Sydney, Australia, September 2012

[14] A. Kühne, A. Klein, A. Loch and M. Hollick, „Opportunistic forwarding in multi-hop OFDMA networks with local CSI," in Proc. 17th International OFDM-Workshop (InOWo'12), Essen, Germany, August 2012

[15] H. Degenhardt, F. Hohmann, A. Klein, „Pilot Transmission Scheme and Robust Filter Design for Non-Regenerative Multi-Pair Two-Way Relaying", In Proceedings of the IEEE PIMRC 2012, Sydney, Australia

[16] H. Degenhardt, A. Klein, „Self-interference aware MIMO filter design for non-regenerative multi-pair two-way relaying", In Proceedings of the IEEE WCNC 2012, Paris, France

[17] E. González-Rodríguez et al. "Impact of Group Delay on Tunable Impedance Matching Networks Based on Barium-Strontium-Titanate Varactors," IEEE ISSSE 2012

[18] Lufei Shen, Klaus Hofmann: "Fully Integratable 4Phase Charge Pump Architecture for High Voltage Applications", Proceedings of the MIXDES 2012 Conference (19th International Conference MIXED Design of Integrated Circuits and Systems), May 25-26 2012, Warsaw, Poland.

[19] Klaus Hofmann, Lufei Shen, Erick GonzalezRodriguez, Holger Maune, Dirk Dahlhaus, Rolf Jakoby: "Fully Integrated High Voltage Charge Pump for Energy-efficient Reconfigurable Multiband RF Transceivers", Proceedings of the IEEE International Telecommunications Energy Conference (INTELEC), Sep 30 - Oct 4 2012, Scottsdale AZ, U.S.A.

[20] Maune et al.: Tunable RF GaN-Power Transistor Implementing Impedance Matching Networks Based on BST Thick Films, EuMIC 2012

[21] Maune et al.: Current Status of Tunable RF Components based on Ferroelectric Thick Films for Power Amplifiers (invited), PA Symposium 2012

[22] Y Cheng A Philipp and M Pesavento "Dynamic Rate Adaptation and Multiuser Downlink Beamforming Using Mixed Integer Conic Programming", In Proc. of EUSIPCO'12, Bucharest, Romania, August, 2012 